\title{
VIABILIDAD DE LAS SOCIEDADES LABORALES EN ECUADOR. UNA APROXIMACIÓN DOCUMENTAL
}

VIABILITY OF LABOR COMPANIES IN ECUADOR. A DOCUMENTARY APPROACH

\section{Mercedes Montiel $^{*}$}

Resumen: Las sociedades laborales se han desarrollado con particular interés en España, conformándose como sociedades anónimas o sociedades de responsabilidad limitada, destacando en sus características que la mayoría del capital social es propiedad de los trabajadores, quienes tienen un $51 \%$ de participación en el mismo, pero con las limitantes de que cada empleado no puede tener más de una tercera parte de lo que conforma el capital de la sociedad, así como que en el caso de romperse la relación laboral, el trabajador debe traspasar sus acciones o cuotas de participación a otros socios por el derecho de preferencia establecido en la sociedad, a fin de mantener las acciones dentro de la misma. El presente trabajo pretende a través de una metodología descriptiva y documental, con una técnica de observación y análisis documental, apoyados en la triangulación y la hermenéutica jurídica, analizar la conformación de las sociedades laborales, sus beneficios y limitaciones, para determinar la conveniencia y pronosticar el impacto de la existencia de sociedades laborales en Ecuador, proponiendo a su vez alternativas acerca de los diversos modos de orientar y formar a los trabajadores y empresarios para su apertura y participación en este tipo de empresas.

Palabras clave: sociedad de capital, trabajador, socio, empresa, empresario

\footnotetext{
* Abogada por la Universidad del Zulia (Venezuela), Magister en Derecho Mercantil, Doctora en Ciencia de la Educación, Post Doctorado en Gerencia Pública y Gobierno de la Universidad Rafael Belloso Chacín (Venezuela), Profesora de Derecho civil, Laboral y Derechos humanos en la Universidad de Los Hemisferios (Quito, Ecuador). mmontiel@uhemisferios.edu.ec; mercedesmatilde1403@hotmail.com
} 
Abstract: Labour Societies have developed a particular interest in Spain, settling as corporations or limited liability companies, highlighting their characteristics that most of the capital is owned by the workers, who have a $51 \%$ stake in the capital, but with the limitations that each employee can not have more than one third of what makes up the capital and in the case of breaking the employment relationship, the worker must transfer their shares or participation to other partners for right of preference established in society, in order to maintain actions within it. For the above stated, the present work aims through a descriptive and documentary methodology with the technique of observation and document analysis, supported by triangulation and legal interpretation, analyze the structure of industrial societies, their benefits and limitations, to determine convenience and forecast the impact of the existence of Labor Societies in Ecuador, proposing his alternative time about the various ways to guide and train workers and entrepreneurs for opening and participation in these businesses.

Keywords: Capital Society, Employee, Partner, Enterprise, Entrepreneur

Sumario. I. Introducción. II. Delimitación del estudio. III. Desarrollo. III.1. Consideración de la normativa de las sociedades laborales. III.2. Regulación de la sociedad laboral en Costa Rica. III.3. Regulación de la sociedad laboral en Argentina. III.4. La figura de la cooperativa. III.5. Análisis de la legislación societaria, considerando la viabilidad de la existencia legal de las sociedades laborales ecuatorianas. IV. Conclusiones. Referencias.

\section{INTRODUCCIÓN}

Las sociedades laborales podríamos pensar que son de reciente data, no siendo así, según Chávez \& Monzón (2007) surgen en Gran Bretaña, a finales del siglo XVIII e inicios del siglo XIX, consolidándose posteriormente, a raíz de la conveniencia o necesidad de interesar a los trabajadores de una empresa en crisis, a ingresar como socios de esta, en base a lo cual, ven como viabilidad económica, transferir la totalidad o gran parte de las acciones de la sociedad a los trabajadores.

Hoy en día, y no siempre por razones de crisis, se presentan sociedades con el mismo espíritu de desarrollo societario o constitutivo, el cual es ingresar trabajadores a la empresa como socios de la misma, o conformarlas desde su inicio con socios-trabajadores. 
Este tipo de sociedad ha tenido mayor repunte en la actualidad en España, no dejando de estar presente en otros países como: Costa Rica, Argentina, México, Colombia, unas como sociedades de capital y en otros lugares se conforman como cooperativas, tal como sucede en México y Colombia. En este trabajo consideraremos las establecidas como sociedades de capital.

Entre los antecedentes de la investigación, se encuentra el estudio efectuado por el Grupo de Estudios Sociales y Económicos del Tercer Sector (GESES) ${ }^{1}$ conformado por el equipo investigador de la Universidad de Zaragoza, integrado por el Dr. Jesús Clemente López, la Dra. Carmen Marcuello Seives y el Dr. Millán Díaz Fonse en el año 2008, titulado "Sociedades Cooperativas y Sociedades Laborales en España, Estudio de su contribución a la creación de empleo y al crecimiento económico" donde determinaron la receptividad y progreso de este tipo de sociedad, la que va en aumento y afianzamiento, como una nueva visión de la participación de los trabajadores en las empresas.

La investigación antes mencionada, reforzó la tesis de la conveniencia de este tipo de sociedad en Ecuador, no solo desde el punto de vista de rentabilidad y beneficio económico, sino de gran influencia social sobre todo en el área de servicios, sumado a la positiva influencia para el individuo al sentir que forma parte y participa en una empresa, la cual también le pertenece.

\section{DELIMITACIÓN DEL ESTUDIO}

Formulación del problema. Todo lo antes indicado da lugar a la siguiente interrogante: ¿Es viable la existencia en Ecuador de la sociedad laboral? Fundamentando así los objetivos de la investigación a continuación:

Objetivo general: analizar la viabilidad de las sociedades laborales en Ecuador.

Objetivos específicos. (i) Caracterizar las sociedades laborales, bajo el marco legal que las contiene. (ii) Establecer la viabilidad de las sociedades laborales, según la legislación ecuatoriana.

Delimitación de la investigación. El área de delimitación de la investigación es Ecuador, por tratarse de la posible existencia de una

\footnotetext{
${ }^{1}$ El equipo investigador de la Universidad de Zaragoza estuvo integrado por los doctores Jesús Clemente López, Carmen Marcuello Seives y Millán Díaz Fonse, estudio de 2008 titulado Sociedades Cooperativas y Sociedades Laborales en España, Estudio de su contribución a la creación de empleo y al crecimiento económico.
} 
sociedad de Capital aplicable en todo el país, efectuada en la ciudad de Quito, desde la Universidad de Los Hemisferios.

Justificación de la investigación. La inquietud sobre esta investigación surge del conocimiento de la existencia de las sociedades laborales, más el análisis de la normativa de compañías del Ecuador, que no limita el capital a las Sociedades de Responsabilidad Limitada (SRL), la cual resulta ser uno de los tipos de sociedades más utilizados para establecer la sociedad laboral. En ordenamientos jurídicos como Venezuela, la SRL sólo puede tener como máximo de capital Bs 2.000.000 (USD 1.800, aprox.) suma esta, ya para 2016, ínfima para constituir y mantener cualquier tipo de sociedad de capital, lo que de inicio cierra la posibilidad en Venezuela de establecer la sociedad laboral bajo este tipo de compañía.

Sumado al anterior incentivo, se obtuvo conocimiento de la existencia de una cooperativa conformada por artesanos ecuatorianos, promocionada por el Párroco de Salinas, el sacerdote salesiano Antonio Polo, quien ayudó a crear cientos de pequeñas empresas cooperativas, que favorecen a la economía local y su población indígena conformadas por los mismos artesanos del lugar, obteniendo por esta valiosa labor en el 2011 el premio internacional de economía social Texemi Cantera. Por ello se consideró que esta posibilidad llevada a sociedades de capital haría más llamativa la inversión para los trabajadores, por la facilidad de créditos y financiamientos, entre otros aspectos.

Metodología de la investigación. La metodología de la presente investigación responde a un tipo documental, descriptivo, con un diseño bibliográfico. La técnica de observación fue documental, apoyándose en la hermenéutica jurídica para el análisis e interpretación de los resultados gracias al constructo teórico efectuado por la investigadora mediante la sistematización de la categoría objeto de estudio. 


\section{DESARROLLO}

\section{III.1. Consideración de la normativa de las sociedades laborales}

Buscando dar respuesta al primer objetivo de la investigación, referido a caracterizar el marco legal de las sociedades laborales, tomando como punto de partida España por considerarla como la abanderada en la actualidad de este tipo de sociedades, se inicia el análisis de los aspectos que describen su naturaleza y condición legal, al presentar dos tipos de constitución: originaria y sobrevenida, lo cual es propio de las sociedades de capital (cfr. Morlés, 2000): (i) la originaria nace como una sociedad laboral de capital; mientras, (ii) la sobrevenida es una sociedad mercantil, que por acuerdo asambleario, deciden sus socios modificar los estatutos, pasando a ser calificada por la autoridad competente, como sociedad laboral. Esto último, no debe verse como una transformación de la sociedad, sino más bien, como un cambio de objeto social (García \& Duque, 2009).

$\mathrm{Su}$ naturaleza societaria implica que la mayoría del capital social es propiedad de los trabajadores, lo que permite una relación laboral estable basada en un contrato indefinido, pero con la salvedad de que los sociostrabajadores no pueden tener más de una tercera parte de acciones o participaciones, y se conformarán mínimo con tres (3) socios, considerando las siguientes excepciones, que se mantienen en un periodo de 36 meses, contados a partir de la conformación de la sociedad: (i) de tratarse de socios de entidades públicas o de participación con mayoría pública, entes no lucrativos o de economía social se permite que la participación no sea más del 50\%; y (ii) en el lapso mencionado, podrá estar conformada la sociedad con dos socios mínimos (cfr. Gómez, 1999).

Es conveniente reparar que, por tratarse estas sociedades del tipo de capital, se les aplica la normativa propia de las sociedades anónimas o de responsabilidad limitada —según decidan los socios sea su tipo societario-, donde uno de sus fines primordiales es el lucro. Sin embargo, al tratarse de una sociedad controlada por los trabajadores en cuanto a participación accionaria y de decisión, su fin último no sólo es la rentabilidad, sino una combinación entre la rentabilidad esperada y el aseguramiento del puesto de trabajo.

Por otro lado, se estima acertado el criterio de los autores García \& Duque $(2009$, p. 2), de considerar este tipo de sociedad como un híbrido entre la sociedad de capital y la cooperativa, porque presenta un cuerpo de sociedad mercantil con espíritu de cooperativa.

Igualmente destaca en la naturaleza de este tipo de sociedad laboral los sujetos que en ella intervienen: (i) Socios-trabajadores, son aquellos 
trabajadores con contrato indefinido, quienes entre todos deben tener más del 50\% del capital social. (ii) Socios-no-trabajadores, se refiere a los que son titulares de acciones o participaciones de la sociedad, pero no tienen control sobre la sociedad; se pueden ver como inversionistas, no trabajadores. (iii) Meros-trabajadores, que tienen un contrato laboral temporal o indefinido, pero no son socios (cfr. Gómez, 1999).

Otro aspecto propio de la sociedad laboral es el referido al tipo de acción o participación. Se tienen las laborales, que son las pertenecientes al socio-trabajador y las generales que corresponden al socio-notrabajador. Ambos tipos tienen el mismo valor nominal y otorgan iguales derechos de representación a sus titulares, sin que puedan darse acciones o participaciones sin derecho a voto.

Los órganos competentes en España, para reconocer - y por lo tanto darle personería jurídica - a la sociedad laboral, son el Ministerio de Empleo y Seguridad Social y el Registro Mercantil (para su inscripción), entes que le otorgan los derechos y obligaciones propios de toda figura societaria.

Luego de creada la sociedad laboral, debe identificarse como anónima o de responsabilidad limitada, indicando que se trata de una sociedad laboral mencionando al final, sus siglas S.A. o S.R.L. según corresponda. Todo de acuerdo con la Ley de Sociedades Laborales del correspondiente país.

Para mantener su condición de sociedad laboral, no se podrá exceder la participación de los socios en relación con el capital social, no deben darse inconvenientes con la reserva especial de la sociedad y naturalmente no debe presentarse decisión asamblearia de extinguir la sociedad laboral (cfr. Gómez, 1999).

En caso de hacerse presentes algunas de las situaciones antes expuestas, el Ministerio de Empleo y Seguridad Social suspenderá la calificación de sociedad laboral, informando de esto al Registro. La pérdida de esta condición implica la suspensión y devolución de ayudas y beneficios públicos que se otorgan a estas sociedades.

Es adecuado considerar que sin darse la extinción de la sociedad, cualquiera de los socios podrá traspasar sus acciones o participaciones, pero siempre respetando el derecho de preferencia estipulado en la Ley de Sociedades Laborales correspondiente al país que reconozca este tipo de sociedad, leyes que serán particularmente tratadas y mencionadas con posterioridad en relación a los países que las contengan en sus ordenamientos jurídicos.

Para hacer uso de este Derecho de Preferencia, el socio propietario informará a la sociedad el número, características y valor de las acciones o 
participaciones que pretenda traspasar, esto con el fin de que la sociedad en un periodo máximo de diez días contados desde que se conoce por la sociedad la decisión del traspaso, comunique al grupo de trabajadoressocios, socios generales y trabajadores-no socios, el traspaso, a objeto de que expongan su interés en adquirir en el periodo de veinte días contados a partir de la notificación, las acciones o participaciones ofrecidas.

El administrador, dará a conocer en el lapso de diez días al socio vendedor la identidad de los interesados, respetando el siguiente orden de prioridad: (i) trabajadores no socios; (ii) socios-trabajadores; (iii) socios de clase general, según su participación en el capital; y, (iv) sociedad, respetando ese orden y condiciones, el socio vendedor de tratarse de varios interesados, traspasará a aquel que escoja. De no mostrar interés ninguno de los antes indicados, el socio dispuesto a vender, podrá traspasar libremente las acciones a persona distinta a las ya mencionadas (cfr. Gómez, 1999).

Antes de continuar, debe distinguirse entre sociedad laboral y sociedad participada, mencionadas en la Ley de Sociedades Laborales y Participadas de España. López (2008, p. 13) destaca que en la sociedad laboral deben cumplirse tres criterios: (i) el capital debe conformarse con aporte mayoritario de los trabajadores; (ii) el contrato laboral de los sociostrabajadores es por tiempo indefinido; y, (iii) individualmente cada socio no puede tener más de la tercera parte del capital social. En cuanto a la sociedad participada, los trabajadores promocionan estrategias que permiten diversas formas de participación, pero se asemejan a las laborales en distintos aspectos, como la participación en el capital y resultados de la sociedad, teniendo derecho a voto e intervención en la toma de decisiones de la sociedad.

Luego de aclarar lo concerniente a estos términos, es conveniente destacar los beneficios que se obtienen con la constitución de este tipo de sociedad, la cual según Chávez \& Monzón (2007) se caracteriza y destaca por la existencia de procesos de decisión democráticos y reparto equitativo de los beneficios, como el aplicar a los socios-trabajadores todo aquello que en el ámbito de empleo y seguridad social se otorga a los trabajadores, sumado a beneficios fiscales para estos trabajadores.

\section{III.2. Regulación de la sociedad laboral en Costa Rica}

Continuando con disposiciones legales sobre sociedades laborales, Costa Rica tiene dentro de su normativa jurídica la Ley 7407 (1994), que rige para este tipo de sociedades. Inicialmente no fue bien acogida, sobre todo por los empresarios. Como dice Cordón (2016), el ser humano es 
renuente a cambiar la forma como viene haciendo las cosas, bien por desconocimiento, otras veces por temor o simplemente por no querer cambiar. Sin embargo, poco a poco se fue asimilando en el país esta nueva forma societaria, con aspectos muy parecidos a la ley española, pero con algunas variantes, que se mencionan destacadamente, como es que solo se da para el tipo de sociedades de capital anónima, el número mínimo de socios es de cuatro.

Igualmente, para legalizar su existencia, debe inscribirse en la Sección Mercantil del Registro Público, previo informe favorable del Departamento de Organizaciones Sociales del Ministerio del Trabajo y Seguridad Social. De no presentarse el respectivo informe en diez días hábiles, se tendrá como autorizada la inscripción. Luego, a más tardar seis meses después de la inscripción en el Registro, se debe convocar a una asamblea general de accionistas para establecer los estatutos internos de funcionamiento.

El art. 5 de la mencionada ley (1994) señala que el capital social debe estar íntegramente pagado en un plazo no mayor de un año desde la constitución de la sociedad. Además, se indica que ninguno de los socios puede poseer acciones que representen más del $25 \%$ del capital social.

Se aprecia en el art. 6 de la ley in comento que el Estado y sus instituciones no pueden participar en el capital de la sociedad anónima laboral cuando se establezcan para otorgar concesiones o contratos con el Estado. No obstante, de otorgarse concesiones o se contrate la prestación de servicios fundamentales, el Estado podrá participar hasta con un $20 \%$ del capital social, por un plazo máximo de tres años.

Tanto en las sociedades laborales de Costa Rica como en las de España, la venta de acciones debe respetar el orden de adquirentes establecido en la norma, donde debe informarse al administrador la decisión de traspasar acciones primero a los trabajadores-no socios, quienes deben responder en el lapso de treinta días a partir de ser notificados; si son varios los interesados se distribuirán en igualdad de condiciones las acciones. De no mostrar interés ninguno este tipo de socios, se notificará a los socios trabajadores, quienes tendrán el mismo lapso y condiciones que los anteriores socios.

Si después de esto, ninguno de estos últimos socios muestra interés en adquirir las acciones ofrecidas, se notificará a los socios-no-trabajadores bajo el mismo plazo y condiciones antes indicadas. De no mostrar interés ninguno de los tipos de socios, la sociedad podrá adquirir las acciones dentro de los diez días de notificada. Por último, si incluso la sociedad no muestra interés en adquirir las acciones, el socio dispuesto a vender podrá ofrecerlas a quien decida y le convenga. Este aspecto se mantiene en los 
diversos ordenamientos jurídicos que contienen la sociedad laboral, basado en el interés de que las acciones o participaciones se mantengan en la misma sociedad.

También señala el art. 25 de la Ley de 1994 que el número de trabajadores que no tenga suscritas y pagadas las acciones no podrá ser superior al $15 \%$ en relación con la totalidad de socios trabajadores.

Al igual que la sociedad laboral de España, si se excede del límite de la posesión de acciones y en el porcentaje del capital social estipulado en la norma, la sociedad perderá su condición de laboral, por lo que el Ministerio de Trabajo y Seguridad Social dará a la sociedad tres meses contados a partir de ser conocida la situación para corregir lo alterado. Caso contrario se dictará una resolución para descalificarla como sociedad laboral.

Siguiendo la conformación de la sociedad laboral de España, cualquier sociedad mercantil, por medio de la reforma de sus estatutos puede acogerse al tipo de sociedad laboral y bajo esta modificación continuar funcionando como sociedad de capital, pero bajo la figura de sociedad laboral.

Según Cano (2008, p. 11), la sociedad laboral busca establecer un modelo de empresa que supere una antigua relación laboral basada en la confrontación u oposición entre dos partes empresario y trabajador, por una relación armoniosa y eficiente entre esos dos puntos centrales de la vinculación.

\section{III.3. Regulación de la sociedad laboral en Argentina}

Este tipo de sociedad surge en Argentina debido a la necesidad de establecer métodos para dar lugar a empleos estables, mediante la participación de los trabajadores en la empresa y el dinamismo impartido a esta relación, creada con el Decreto 1406/2001.

En su conformación y naturaleza como sociedad laboral, presenta similitud con los países antes indicados. Sin embargo, muestra las siguientes variantes: (i) las entidades públicas o personas jurídicas pueden poseer hasta un 49\% del capital de la sociedad; y, (ii) pueden acogerse a esta diferente figura societaria, tanto las sociedades anónimas como las sociedades de responsabilidad limitada, distinguiéndose la primera con las siglas S.A.L. y las segundas con las siglas S.L.L., indicando su carácter laboral.

Para constituirse como sociedad laboral se debe obtener la calificación por parte del Registro de Sociedades Laborales del Ministerio del Trabajo y Asuntos Sociales y luego por el Registro Mercantil. 
De lo desarrollado hasta ahora, puede apreciarse que, en aquellos países donde se han establecido, las sociedades laborales presentan similitudes y variantes, más deben resaltarse las características centrales que la identifican como sociedad laboral, presentes en todas ellas, como son las siguientes: (i) Deben conformarse por personas naturales, donde la mayoría sean trabajadores por tiempo indefinido. (ii) El control de la sociedad debe recaer en manos de los socios-trabajadores. (iii) El porcentaje de trabajadores-no-socios es limitado, porque los sociostrabajadores cumplen la gran mayoría de las actividades y gestiones de la sociedad, lo que permite identificar a la sociedad como empresa autogestionaria. (iv) Se controla legalmente que las acciones y participaciones al disponerse, en lo posible, queden en la misma sociedad laboral. Todo esto, como dice Felice (2010, p. 3), debe estar indicado en los estatutos, los cuales recogen las normas básicas de la sociedad laboral.

\section{III.4. La figura de la cooperativa}

Aun cuando no se desarrolla en el presente trabajo la figura de cooperativas laborales, conviene indicar que en países como Colombia y México se ha establecido una relación bastante bien llevada entre trabajadores y entidad societaria de cooperativa, pero que a criterio de la autora, no aprovecha los beneficios económicos y de posesión propia de la sociedad de capital, porque se tratan de organizaciones sin fines de lucro pertenecientes al sector solidario y no emprendedor de la economía, donde sus miembros sólo contribuyen al sostenimiento económico de la cooperativa, sumado a su aporte de trabajo.

Por lo tanto, en este tipo de figura jurídica no se habla de sueldo o salario propio de toda remuneración laboral, sino de compensación al trabajador; no se reconoce la existencia de una relación laboral, con todos los derechos y obligaciones que esta conlleva, sino de una mutua colaboración, todo lo cual limita el incentivo del trabajador a darse por algo que le brinda estabilidad y futuros beneficios económicos y de crecimiento personal (cfr. Farías, 2003, p. 3).

\section{III.5. Análisis de la legislación societaria, considerando la viabilidad de la existencia legal de las sociedades laborales ecuatorianas}

En relación al segundo objetivo de la investigación, y efectuando el debido análisis y consideración de las disposiciones legales del Ecuador en materia de sociedades, se aprecia que la Constitución de la República del Ecuador (2008) establece en su art. 213 que la actividad de las sociedades mercantiles se encuentra regulada por la Superintendencia de Compañías, 
órgano encargado de supervisar las actividades de las compañías o sociedades de capital, por lo que aún no mencionando de modo concreto a las sociedades, reconoce su existencia al indicar la entidad encargada de su control y regulación.

Como se ha expuesto en el desarrollo del presente trabajo, la sociedad laboral puede acogerse a la figura jurídica de sociedad de responsabilidad limitada o de sociedad anónima. Ambas sociedades para su existencia requieren de la manifestación de voluntad de las partes que las conforman, pero para adquirir personalidad jurídica deben cumplir con la solemnidad de su inscripción en el Registro Mercantil.

Ahora bien, como indican Ospina \& Ospina (2014), ni la solemnidad suple la voluntad, ni la voluntad reemplaza la solemnidad en los contratos, sino que se complementan. A más de la voluntad de las partes para asociarse y del cumplimiento de las solemnidades, debe considerarse la libertad de los socios para realizar el contrato societario, basado en su capacidad legal, tal como lo expone el art. 1462 del Código Civil ecuatoriano, al expresar que «toda persona es legalmente capaz, excepto las que la ley declara incapaces». Esto puede extenderse, como indica Larrea (2008, p. 46), al término «ser hábiles para contratar», que no es otra cosa que la capacidad jurídica para contratar, es decir actuando, ejerciendo efectivamente esa capacidad.

Luego de indicar los aspectos fundamentales para que se configure el contrato societario, se pasa a considerar lo concerniente a lo expuesto en los arts. 93 y 94 de la Ley de Compañías de Ecuador, relacionados con las actividades propias de estas sociedades, las cuales son netamente mercantiles, lo que no da carácter de comerciante a sus integrantes. Ellas pueden realizar todo tipo de acto civil, de comercio o mercantil permitido por la ley, salvo ejecutar actividades relativas a operaciones de banco, seguros, capitalizaciones y ahorro. Como se observa, lo exigido por la norma es perfectamente aplicable a la sociedad laboral, conformada bajo cualquiera de las dos figuras societarias antes expuestas.

Otro aspecto a no pasar por alto, es el contenido en el art. 95 de la mencionada Ley de Compañías, que establece que este tipo de sociedad no podrá funcionar si sus socios exceden al número de quince: de excederse debe disolverse o transformarse. Lo indicado en este artículo no va en contra de las exigencias de una sociedad laboral, ya que se puede constituir a partir de dos o tres socios y de ser más de quince, no se conformaría como sociedad de responsabilidad limitada sino como sociedad anónima, que como antes indicamos, son los dos tipos de figuras societarias que puede tomar la sociedad laboral. 
En cuanto al art. 96 de la Ley mencionada, la existencia de esta sociedad se toma desde el momento de su inscripción por ante el Registro Mercantil, siendo esta la misma condicionante para las sociedades laborales. Como se aprecia, la normativa societaria ecuatoriana perfectamente permite la existencia de la sociedad laboral sin alterar el contexto de la norma.

Igualmente resultaría conveniente considerar para estas sociedades en Ecuador, la exoneración fiscal, como se da en Costa Rica, donde no paga impuesto la Sociedad Laboral, en base al Fallo constitucional de los arts. 1, 3 y 5 y la Ley 9024 del año 2016. Sin embargo, sería adecuado analizar el beneficio para las partes intervinientes de un pago, como ocurre en España y Argentina. En España goza de ciertos beneficios fiscales, contenidos en el Real Decreto 2696 de 1986, pero cancela impuestos e IVA. En Argentina la carga impositiva para las Sociedades Laborales es bastante alta, al igual que la carga impositiva del resto de las sociedades de ese país.

Este aspecto tributario por su extensión y detalle de análisis, no se desarrolla en el presente trabajo. Debería ser objeto de un estudio posterior.

\section{CONCLUSIONES}

Dando respuesta a los objetivos de la investigación desarrollados hasta el momento, y haciendo referencia al marco legal de las sociedades laborales, planteado como el primer objetivo del presente trabajo, se observa, la conveniencia y efectividad de la sociedad laboral, bajo la figura de sociedad de capital.

Igualmente, se aprecia la unión y confusión de la figura empresariotrabajador, destacándose los principios de complementariedad y apoyo en estas dos posiciones, lo que aprovecha no sólo a la empresa sino a los involucrados en ella.

Este tipo de sociedad redunda en beneficios no sólo para sus partícipes vistos como un todo, sino para el entorno social, económico y empresarial.

Del análisis en el ordenamiento jurídico ecuatoriano, dando respuesta al segundo objetivo del presente estudio, sobre la conformación de figura societaria se confirmó que la sociedad laboral tiene alguna cabida dentro del ordenamiento jurídico ecuatoriano, bien como sociedad anónima o sociedad de responsabilidad limitada.

Ahora bien, la hipótesis no puede concretarse hasta tanto se determine la aceptación de este tipo de sociedad dentro de la cultura 
empresarial ecuatoriana, lo cual lo vemos algo complejo, ya que el empresario ecuatoriano parece concebir la empresa como algo que debe ser manejada y controlada por él, apreciándose en general poca propensión a permitir al trabajador su participación en la empresa, más allá de su posición de trabajador.

Sumado a la anterior percepción, sería necesaria la aplicación de instrumentos de investigación que permitan medir la aptitud y actitud del empresario para la aceptación de esta figura societaria distinta, para lo cual podría ser necesario formar y preparar tanto al empresario como al trabajador para lograr un cambio de visión al respecto. 


\section{REFERENCIAS}

Cano, A. (2008). Algunas reflexiones tópicas al hilo de una singular y necesaria propuesta de reforma. Revista Jurídica de la Economía Social y cooperativa, 19, pp. 159171.

Chávez, R. \& Monzón J. (2007). La Economía Social en la Unión Europea. Informe de la CIRIEC, sobre Economía Pública, social y cooperativa. Bruselas: Editorial Ciriec.

Cordón, J. (V-1994). La sociedad laboral en Costa Rica. San José (Costa Rica): Periódico La Nación.

Farías, C. (2003). Mapeando al Cooperativismo. Rosario: UNR.

Felice, C. (2010). Que es una sociedad laboral. Argentina. En https://utta2010.wordpress.com/el-trabajo-constituye-el-lazo-de-union-entre-elhombre-y-la-sociedad/\%C2\%BFque-es-una-sociedad-anonima-laboral/ (recuperado el 20-VII-2017).

García, M. \& Duque, J. (2009). La interesante figura de las sociedades laborales. Cayapa, revista Venezolana de Economía Social, 18(9), pp. 75-92.

Gómez Calero, J. (1999). Las sociedades laborales. Granada: Comares.

Larrea, J. (2008). Manual elemental de Derecho Civil del Ecuador. Contratos. Quito: CEP.

López, G. (2008). Las sociedades laborales desde el Derecho del Trabajo, cuestiones centrales y propuesta de reforma. Revista Jurídica de la Economía Social y cooperativa, 19 , pp. 251-262.

Morlés, A. (2000). Curso de Derecho Mercantil. Caracas: Universidad Católica Andrés Bello.

Ospina, G. \& Ospina E. (2014). Teoría General del Contrato y del Negocio Jurídico. Bogotá: Temis.

\section{Normativa}

Argentina, Decreto 1406 de sociedades laborales (2001).

Costa Rica, Ley 7407 de sociedades laborales (1994).

Ecuador, Ley de Compañías de Ecuador (1999).

Constitución de la Republica de Ecuador (2008).

Código Civil ecuatoriano (2016). 